services, demonstrating that fundamental nursing skills such as compassion, communication, flexibility and kindness are transferable, and the contributions of Children's Nurses in these and other fields were important and valued by others.

\section{DOCUMENTATION OF CO-MORBIDITIES IN PATIENTS HAVING A SURGICAL PROCEDURE}

${ }^{1}$ Nikita Mehtani, ${ }^{2}$ Kailas Bhandarkar, Dhanya Mullassery. ${ }^{1}$ Imperial College Healthcare NHS Trust; ${ }^{2}$ Great Ormond Street Hospital

10.1136/archdischild-2020-gosh.63

Children with multiple comorbidities are typically referred to GOSH as GOSH is a tertiary centre for management of children with complex medical and surgical conditions.

Often these children require multiple admissions. This requires several patient encounters, which raises opportunity for missing important clinical information. The team re-audited how well, patient co-morbidities were documented on EPIC (electronic patient record). This is not only useful for the clinical team for accuracy of information but also has financial implications for the hospital.

A total of 71 children underwent a general surgical procedure over a 4-week period. Their inpatient notes were analysed, specifically Pre-op clerking (Admission note), Operation note \& Discharge summary. Of these, only 16 patients did not have any associated comorbidity. The findings show 92.9\% (Pre-op clerking), 35.2\% (Op-note) \& 57.7\% (Discharge Summary) had accurate documentation of comorbidities. Only $1.8 \%$ of patients had missed comorbidities post EPIC compared to $29 \%$ pre-EPIC. This echoes that EPIC has made it effortless for coding team to record clinical information accurately \& has important financial implications for the trust.

\section{WALKER WARBURG SYNDROME (WWS) WITH ISPD GENETIC MUTATION- A CASE REPORT}

${ }^{1}$ Premala Muthukumarasamy, ${ }^{1}$ Haroon Mansoori, ${ }^{2}$ Ahmed Albialy, ${ }^{1}$ Jenn Tan, ${ }^{1}$ Kerry Brown, ${ }^{1}$ Robert Henderson, ${ }^{1}$ Bridget Callaghan, 'Sian Pincott, ${ }^{1}$ Adnan Manzur. 'Great Ormond Street Hospital; ${ }^{2}$ North West Anglia NHS trust

\subsection{6/archdischild-2020-gosh.64}

Background Walker Warburg dystroglycanopathy is the most severe subtype of congenital muscular dystrophies. It is characterised by four consistent features -cobble stone lissencephaly, cerebellar malformation, retinal abnormalities and muscular dystrophy. The incidence is estimated at 1.2 per 100,000 live births and the life-span in these children is around 3 years. There are more than fifteen causative genes and transmission is autosomal recessive.

Case We describe a boy, born in Kuwait to parents of a consanguineous marriage, premature at 34 weeks gestation with hypotonia, antenatal hydrocephalus and severe eye abnormalities. An MRI brain done at the referring hospital showed cobblestone lissencephaly and severe ventricular dilatation. He was transferred to our centre at corrected age 38 weeks for neurosurgical and medical management. Clinical examination showed central hypotonia with a lack of purposeful movements, macrocephaly from hydrocephalus, left eye buphthalmos and congenital glaucoma, a blind microphthalmic right eye, hearing impairment and bilateral medullary nephrocalcinosis. The creatinine kinase was elevated at 3996U/l. A genetic panel for muscular dystrophy done here detected a homozygous ISPD c.1186G $>$ T p. (Gly396Ter) pathogenic sequence variant, confirming ISPDrelated congenital muscular dystrophy. Complications included cerebrospinal fluid leak post ventriculoperitoneal (VP) shunt insertion, recurrent aspiration pneumonia and urinary tract infections. Of note, he developed hypothermia as a side effect from Timolol eye drops as part of his treatment for glaucoma. He underwent a fundoplication gastrostomy ;slowly tolerating feeds with anti-reflux medications, breathing comfortably in air and cooing in response to parents' handling. Within a comprehensive multidisciplinary team effort, lung protective strategies, regular occupational and physiotherapy, he made a good recovery. He was successfully repatriated home with good parental care at corrected age 7 months

Conclusion Prognosis may be poor in WWS, however it is most ethical to provide holistic care, optimise the quality of life and direct genetic counselling.

\section{MANAGEMENT OF EARLY-ONSET SCOLIOSIS IN PATIENTS WITH PRADER WILLI SYNDROME WITH MAGNETIC GROWTH RODS - A CASE SERIES REVIEW}

Henry Bowyer, Amir Amiri, Mark Harris. Great Ormond Street Hospital

\subsection{6/archdischild-2020-gosh.65}

Background Prader Willi syndrome (PWS) is a rare genetic disorder characterised by developmental delay and hyperphagia. It is strongly associated with scoliosis, largely attributed to hypotonia and ligamentous laxity. Management of scoliosis in these patients can be challenging due to early onset of disease, higher BMI, poor bone quality and poor brace compliance. Skin picking behaviours may also have a significant impact on poor healing. Magnetic Expansion Control growing rods (MAGEC) have become the surgical management of choice in some patients with progressive early-onset scoliosis, however there have been no published reports on their use in the PWS population. Here we report our experience of treating children with PWS with MAGEC rods.

Methods A retrospective review of children with PWS who underwent MAGEC rod insertion between March 2010 to March 2020 at a quaternary paediatric hospital. Spinal column distraction was measured between the proximal and distal vertebrae in which fixations were inserted.

Results 4 patients with PWS had MAGEC rod inserted during the study period. All had undergone MRI imaging prior to surgery. Median age at primary operation was 5.5 years. All patients were overweight at time of primary operation (median BMI 94.5th centile).

A total of 11 MAGEC rods were used of which 5 had reported instrumentation failures (45\%). These included 2 pin fractures and 3 proximal construct pull-outs. 2 of the patients developed post-operative infection requiring surgical intervention with 1 undergoing removal of metalwork.

Overall a median of $4.3 \mathrm{~mm}(\mathrm{IQR}=1.1-25.3)$ of spinal distraction was achieved either prior to definitive spinal fusion or at last follow-up with median distraction per year of MAGEC of $1.1 \mathrm{~mm}(\mathrm{IQR}=0.5-5.1)$. 